\title{
A NOTE ON THE LINEAR SYSTEMS ON THE PROJECTIVE BUNDLES OVER ABELIAN VARIETIES
}

\author{
LEI ZHANG
}

(Communicated by Lev Borisov)

\begin{abstract}
It is well known that for an ample line bundle $L$ on an Abelian variety $A$, the linear system $|2 L|$ is base point free and $3 L$ is very ample; moreover, the map defined by the linear system $|2 L|$ is well understood (cf. Theorem 1.1). In this paper we generalize this classical result and give a new proof using the theory developed by Pareschi and Popa in 2011 (cf. Theorem 1.2).
\end{abstract}

\section{INTRODUCTION}

Conventions. All the varieties are assumed over $\mathbb{C}$. For a variety $X$ and a vector bundle $E$ on it, $\mathbb{P}_{X}(E)$ is defined as $\operatorname{Proj}_{\mathcal{O}_{Y}}\left(\bigoplus_{k} S^{k}\left((E)^{*}\right)\right)$ and $\mathcal{O}_{\mathbb{P}_{X}(E)}(1)$ denotes the anti-tautological bundle. Let $f: X \rightarrow Y$ be a morphism between two smooth projective varieties, denote by $D^{b}(X)$ and $D^{b}(Y)$ their bounded derived categories of coherent sheaves, and by $R f_{*}$ and $L f^{*}$ the derived functors of $f_{*}$ and $f^{*}$ respectively. For $E \in D^{b}(X), E^{*}$ denotes its dual $R \mathcal{H} \operatorname{Hom}\left(E, \mathcal{O}_{X}\right)$, and we say that $E$ is a sheaf if it is quasi-isomorphic to a sheaf in $D^{b}(X)$. If $X=X_{1} \times X_{2} \times \ldots \times X_{r}$ denotes the product of $r$ varieties, then $p_{i}$ denotes the projection from $X$ to the $i$-th factor $X_{i}$. If $A$ denotes an Abelian variety, then $\hat{A}$ denotes its dual $\operatorname{Pic}^{0}(A)$, $\mathcal{P}$ denotes the Poincaré line bundle on $A \times \hat{A}$, and the Fourier-Mukai transform $R \Phi_{\mathcal{P}}: D^{b}(A) \rightarrow D^{b}(\hat{A})$ w.r.t. $\mathcal{P}$ is defined as

$$
R \Phi_{\mathcal{P}}(\mathcal{F}):=R\left(p_{2}\right)_{*}\left(L p_{1}^{*} \mathcal{F} \otimes \mathcal{P}\right) ;
$$

similarly $R \Psi_{\mathcal{P}}: D^{b}(\hat{A}) \rightarrow D^{b}(A)$ is defined as

$$
R \Psi_{\mathcal{P}}(\mathcal{F}):=R\left(p_{1}\right)_{*}\left(L p_{2}^{*} \mathcal{F} \otimes \mathcal{P}\right) .
$$

For a sheaf $\mathcal{F}$ on an Abelian variety $A$, if $R \Phi_{\mathcal{P}}(\mathcal{F}) \cong R^{0} \Phi_{\mathcal{P}}(\mathcal{F})$, we say $\mathcal{F}$ satisfies $I T^{0}$. If $a: X \rightarrow A$ denotes a map to an Abelian variety, then $\mathcal{P}_{a}:=\left(a \times i d_{\hat{A}}\right)^{*} \mathcal{P}$, and for $\mathcal{F} \in D^{b}(X), R \Phi_{\mathcal{P}_{a}}(\mathcal{F})$ is defined similarly; if $\alpha \in \hat{A}$, we often denote the line bundle $a^{*} \alpha \in P i c^{0}(X)$ by $\alpha$ for simplicity.

For the linear systems over an Abelian variety, the following classical results are well known.

Theorem 1.1. Let $A$ be an Abelian variety and $L$ an ample line bundle on it. Then $3 L$ is very ample, $|2 L|$ is base point free, and if $2 L$ is not very ample, then

(i) $A=A_{1} \times A_{2}$ and $L \cong L_{1} \otimes L_{2}$, where $L_{i}$ is a line bundle on $A_{i}$ and at least one of $\left(A_{i}, L_{i}\right)$ is a principal polarization $([12],[])$.

Received by the editors March 18, 2012 and, in revised form, July 27, 2012.

2010 Mathematics Subject Classification. Primary 14E05; Secondary 14K99.

Key words and phrases. Abelian varieties, linear system, birational maps. 
(ii) Moreover, if $A$ is simple and $(A, L)$ is a principal polarization, then $L$ is symmetric up to translation, and the map $\phi$ defined by $|2 L|$ coincides with the quotient map $A \rightarrow A /(-1)_{A}$ ([1], Sections 4.5, 4.8).

In this paper, as a generalization, we prove

Theorem 1.2. Let $A$ be an Abelian variety, $E$ an $I T^{0}$ vector bundle on it, and $P=$ $\mathbb{P}_{A}\left(E^{*}\right)$ with an anti-tautological line bundle $\mathcal{O}_{P}(1)$. Then $\mathcal{O}_{P}(3)$ is very ample, and the linear system $\left|\mathcal{O}_{P}(2)\right|$ is base point free and hence defines a morphism $\phi$. Moreover, $\phi$ is not birational if and only if $A \cong A_{1} \times A_{2}$, and $E \cong L_{1} \otimes E_{2}$, where $L_{1}$ is a line bundle on $A_{1}$ and $E_{2}$ is a vector bundle on $A_{2}$ such that either $\chi\left(A_{1}, L_{1}\right)=1$ or $\chi\left(A_{2}, E_{2}\right)=1$.

In particular, if $A$ is simple, then $\phi$ is not birational if and only if, up to translation, $E$ is a $(-1)_{A}$-invariant sheaf satisfying one of the following:

(i) $\chi(A, E)=1$, i.e., $R \Phi_{\mathcal{P}}(E) \cong \mathcal{O}_{\hat{A}}(-\hat{D})$ where $\hat{D}$ is an ample divisor on $\hat{A}$, and then $\operatorname{deg}(\phi)=2$ when $\operatorname{dim}(A) \geq 2$ and $\operatorname{deg}(\phi)=2^{\operatorname{rank}(E)}$ when $\operatorname{dim}(A)=1$;

(ii) $E \cong \bigoplus^{n} \mathcal{O}_{A}(L)$ where $(A, L)$ is a principal polarization, and then $\operatorname{deg}(\phi)=$ 2 .

Meanwhile there exists an involution $\sigma$ on $P$ such that $\phi$ factors through the quotient map $P \rightarrow P /(\sigma)$, which fits into the following commutative diagram:

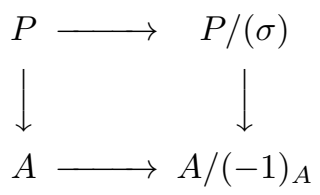

Remark 1.3. Comparing the two properties $I T^{0}$ and ampleness, they are equivalent for a line bundle on an Abelian variety, and for a vector bundle, Debarre proved that the property $I T^{0}$ implies ampleness $([\underline{5}]$ ).

Remark 1.4. The classical proof of Theorem 1.1 is beautiful but very long. Here we not only generalize those classical results but also provide a brief proof thanks to the theory developed by Pareschi and Popa ([11, [10). It is key to consider the case when $A$ is simple, and when $A$ is not simple, with the help of Theorem 3.6 and 3.5 in Section 3 , we can also understand the morphism $\phi$ well (cf. Section 3.4).

\section{Definitions And technicAl Results}

In this section, we list some definitions and results which will be used in this paper.

Theorem 2.1 ([8], Thm. 2.2). Let $A$ be an Abelian variety of dimension d. Then

$$
R \Psi_{\mathcal{P}} \circ R \Phi_{\mathcal{P}}=(-1)_{A}^{*}[-d], R \Phi_{\mathcal{P}} \circ R \Psi_{\mathcal{P}}=(-1)_{\hat{A}}^{*}[-d]
$$

and

$$
R \Phi_{\mathcal{P}} \circ(-1)_{A}^{*} \cong(-1)_{\hat{A}}^{*} \circ R \Phi_{\mathcal{P}}, R \Psi_{\mathcal{P}} \circ(-1)_{\hat{A}}^{*} \cong(-1)_{A}^{*} \circ R \Psi_{\mathcal{P}}
$$

As a corollary we have

Corollary 2.2. Let $A$ be an Abelian variety of dimension d, E an object on $D^{b}(A)$, and $F=R \Phi_{\mathcal{P}}(E)^{*}$. Then

$$
R \Phi_{\mathcal{P}}(E)=F^{*}, R \Psi_{\mathcal{P}}(F)=E^{*} .
$$


Proof. We need to show the second equation. Let $p_{1}$ and $p_{2}$ be the projections from $A \times \hat{A}$ to $A$ and $\hat{A}$ respectively. Then we have

$$
\begin{aligned}
R \Psi_{\mathcal{P}}(F)= & R\left(p_{1}\right)_{*}\left(\operatorname{Lp} p_{2}^{*} R \mathcal{H} \operatorname{tom}\left(R \Phi_{\mathcal{P}}(E), \mathcal{O}_{\hat{A}}\right) \otimes \mathcal{P}\right) \\
= & R\left(p_{1}\right)_{*}\left(R \mathcal{H o m}\left(\operatorname{Lp} p_{2}^{*} R \Phi_{\mathcal{P}}(E), \mathcal{O}_{A \times \hat{A}}\right) \otimes \mathcal{P}\right) \\
= & R\left(p_{1}\right)_{*} R \mathcal{H o m}\left(\operatorname{Lp}_{2}^{*} R \Phi_{\mathcal{P}}(E), \mathcal{P}\right) \\
= & R\left(p_{1}\right)_{*} R \mathcal{H o m}\left(\operatorname{Lp}_{2}^{*} R \Phi_{\mathcal{P}}(E) \otimes \mathcal{P}^{-1}, \mathcal{O}_{A \times \hat{A}}\right) \\
= & R \mathcal{H o m}\left(R\left(p_{1}\right)_{*}\left(\operatorname{Lp}_{2}^{*} R \Phi_{\mathcal{P}}(E) \otimes \mathcal{P}^{-1}\right), \mathcal{O}_{A}[-d]\right) \\
& \cdots \operatorname{by} \operatorname{Grothendieck~duality} \\
= & R \mathcal{H o m}\left(R \Psi_{\mathcal{P}-1} R \Phi_{\mathcal{P}}(E), \mathcal{O}_{A}[-d]\right) \\
= & R \mathcal{H o m}\left((-1)_{A}^{*} R \Psi_{\mathcal{P}} R \Phi_{\mathcal{P}}(E), \mathcal{O}_{A}[-d]\right) \cdots \text { by } R \Psi_{\mathcal{P}^{-1}}=(-1)_{A}^{*} R \Psi_{\mathcal{P}} \\
= & R \mathcal{H o m}\left(E[-d], \mathcal{O}_{A}[-d]\right)=E^{*} \cdots \text { by Theorem } 2.1
\end{aligned}
$$

Definition 2.3 ([10], Def. 2.1, 2.8, 2.10; 4], Def. 2.6). Given a coherent sheaf $\mathcal{F}$ on an Abelian variety $A$, its $i$-th cohomological support locus is defined as

$$
V^{i}(\mathcal{F}):=\left\{\alpha \in \operatorname{Pic}^{0}(A) \mid h^{i}(\mathcal{F} \otimes \alpha)>0\right\} .
$$

The number $g v(\mathcal{F}):=\min _{i>0}\left\{\operatorname{codim}_{P i c^{0}(A)} V^{i}(\mathcal{F})-i\right\}$ is called the generic vanishing index of $\mathcal{F}$, and we say $\mathcal{F}$ is a $G V$-sheaf (resp. $M$-regular sheaf) if $g v(\mathcal{F}) \geq 0$ (resp. $>0$ ).

Let $X$ be an irregular variety equipped with a morphism to an Abelian variety $a: X \rightarrow A$. Let $\mathcal{F}$ be a sheaf on $X$; its $i$-th cohomological support locus w.r.t. $a$ is defined as

$$
V^{i}(\mathcal{F}, a):=\left\{\alpha \in \operatorname{Pic}^{0}(A) \mid h^{i}\left(X, \mathcal{F} \otimes\left(a^{*} \alpha\right)\right)>0\right\} .
$$

We say $\mathcal{F}$ is full w.r.t. the map $a$ if $V^{0}(\mathcal{F}, a)=\hat{A}$, and is continuously globally generated (CGG) w.r.t. $a$ if the sum of the evaluation maps

$$
e v_{U}: \bigoplus_{\alpha \in U} H^{0}(\mathcal{F} \otimes \alpha) \otimes\left(\alpha^{-1}\right) \rightarrow \mathcal{F}
$$

is surjective for any open set $U \subset \hat{A}$.

Let's recall the following results due to Pareschi and Popa.

Proposition 2.4 ([10, Prop. 3.1). Let $\mathcal{F}$ be a $G V$-sheaf and $H$ a locally free $I T^{0}$ sheaf on an Abelian variety $A$. Then $\mathcal{F} \otimes H$ satisfies $I T^{0}$.

Proposition 2.5 ([10], Cor. 5.3). An M-regular sheaf on an Abelian variety is $C G G$.

Proposition 2.6 (11], Prop. 2.12). Let $X$ be an irregular variety equipped with a morphism to an Abelian variety $a: X \rightarrow A$. Let $F$ be a coherent sheaf and $L$ a line bundle on $X$. Suppose that both $F$ and $L$ are continuously globally generated w.r.t. a. Then $F \otimes L \otimes \alpha$ is globally generated for all $\alpha \in \operatorname{Pic}^{0}(A)$.

Theorem 2.7 ([11], Thm. 5.1). Let $F$ be a $G V$-sheaf on an Abelian variety $A$. Then the following conditions are equivalent:

(a) F is M-regular. 
(b) For every locally free $I T^{0}$ sheaf $H$ on A, and for every Zariski open set $U \subset \hat{A}$, the sum of the multiplication of maps of global sections

$$
\bigoplus_{\alpha \in U} H^{0}(A, F \otimes \alpha) \otimes H^{0}\left(A, H \otimes \alpha^{-1}\right) \rightarrow H^{0}(A, F \otimes H)
$$

is surjective.

Remark 2.8. Modifying the proof of Theorem 5.1 in [11, we can show that for a $\mathrm{GV}$-sheaf $F$ and a locally free $I T^{0}$ sheaf $H$ on $A$, the sum of the multiplication of maps of global sections

$$
\bigoplus_{\alpha \in \hat{A}} H^{0}(A, F \otimes \alpha) \otimes H^{0}\left(A, H \otimes \alpha^{-1}\right) \rightarrow H^{0}(A, F \otimes H)
$$

is surjective.

As a corollary of the theorem above, we get an interesting result.

Lemma 2.9. Let $E$ be an $I T^{0}$ vector bundle on an Abelian variety A. Suppose that $\chi(A, E)=1$ and that $E$ is a $(-1)_{A}$-invariant sheaf. Let $\varphi_{E}: E \rightarrow(-1)_{A}^{*} E$ be the corresponding isomorphism. Then naturally $\varphi_{E} \otimes \varphi_{E}: E \otimes E \rightarrow(-1)_{A}^{*}(E \otimes E)$ shows that $E \otimes E$ is a $(-1)_{A}$-invariant sheaf; thus $(-1)_{A}$ induces an action $(-1)_{A}^{*}$ on $H^{0}(A, E \otimes E)$. Moreover, if we denote its invariant (resp. anti-invariant) subspace by $H^{0}(A, E \otimes E)^{+}\left(r e s p . H^{0}(A, E \otimes E)^{-}\right)$, then we have

$$
H^{0}\left(A, S^{2} E\right) \cong H^{0}(A, E \otimes E)^{+} \text {and } H^{0}\left(A, \bigwedge^{2} E\right) \cong H^{0}(A, E \otimes E)^{-} .
$$

Proof. Since $h^{0}(A, E \otimes \alpha)=1$, we can assume $H^{0}(A, E \otimes \alpha)=\operatorname{span}\left\{e_{\alpha}\right\}$ for every $\alpha \in \hat{A}$. Applying Theorem 2.7. $H^{0}(A, E \otimes E)$ is spanned by $\left\{e_{\alpha} \otimes e_{\alpha^{-1}}\right\}_{\alpha \in \hat{A}}$, and hence $H^{0}\left(A, S^{2} E\right)$ (resp. $\left.H^{0}\left(A, \bigwedge^{2} E\right)\right)$ is spanned by $\left\{e_{\alpha} \otimes e_{\alpha^{-1}}+e_{\alpha} \otimes e_{\alpha^{-1}}\right\}_{\alpha \in \hat{A}}$ (resp. $\left.\left\{e_{\alpha} \otimes e_{\alpha^{-1}}-e_{\alpha} \otimes e_{\alpha^{-1}}\right\}_{\alpha \in \hat{A}}\right)$. Since $(-1)_{A}^{*} e_{\alpha}$ is a section of $(-1)_{A}^{*}(E \otimes \alpha) \cong$ $E \otimes \alpha^{-1}$ and $H^{0}\left(A, E \otimes \alpha^{-1}\right)$ is spanned by $e_{\alpha^{-1}}$, we can write $(-1)_{A}^{*} e_{\alpha}=c e_{\alpha^{-1}}$ with $c \neq 0$, and then $(-1)_{A}^{*} e_{\alpha^{-1}}=\frac{1}{c} e_{\alpha}$, hence $(-1)_{A}^{*}\left(e_{\alpha} \otimes e_{\alpha^{-1}}\right)=e_{\alpha^{-1}} \otimes e_{\alpha}$. Finally we find that

$$
(-1)_{A}^{*}\left(e_{\alpha} \otimes e_{\alpha^{-1}}+e_{\alpha} \otimes e_{\alpha^{-1}}\right)=e_{\alpha} \otimes e_{\alpha^{-1}}+e_{\alpha} \otimes e_{\alpha^{-1}}
$$

and

$$
(-1)_{A}^{*}\left(e_{\alpha} \otimes e_{\alpha^{-1}}-e_{\alpha} \otimes e_{\alpha^{-1}}\right)=-\left(e_{\alpha} \otimes e_{\alpha^{-1}}-e_{\alpha} \otimes e_{\alpha^{-1}}\right) .
$$

So we are done.

Here we make a simple but very useful remark, which is probably well known to experts, but we are not able to find a reference.

Theorem 2.10. Let $X$ and $Y$ be two normal projective varieties, and $\mathcal{L}$ a line bundle on $X \times Y$. Assume $E=\left(p_{2}\right)_{*} \mathcal{L}$ is a vector bundle and put $P=\mathbb{P}_{Y}(E)$. Then there exists an open set $U \subset Y$ such that $\mathbb{P}_{U}(E)$ parametrizes the divisors in $\left|\mathcal{L}_{y}\right|, y \in U$. Correspondingly we get the universal family $\mathcal{D}_{U} \subset X \times U \rightarrow U$. Denote the closure of $\mathcal{D}_{U}$ in $X \times P$ by $\mathcal{D}$, which is embedded in $X \times P$ as a divisor. Then we have

$$
\mathcal{D} \equiv p^{*} \mathcal{L} \otimes q^{*} \mathcal{O}_{P}(1)
$$

where $p, q$ denote the two projections $p: X \times P \rightarrow X \times Y, q: X \times P \rightarrow P$. 
Proof. Let $\left\{U_{\alpha}\right\}_{\alpha \in I}$ be an affine cover of $Y$. Assume $E$ is of rank $n$ and $E\left(U_{\alpha}\right)=$ $\mathcal{O}_{Y}\left(U_{\alpha}\right)\left(s_{\alpha}^{1}, s_{\alpha}^{2}, \ldots, s_{\alpha}^{n}\right)$ where $s_{\alpha}^{i} \in H^{0}\left(X \times U_{\alpha}, \mathcal{L}\right) \cong E\left(U_{\alpha}\right)$, and the divisors $\left(s_{\alpha}^{i}\right)$ have no common vertical components (where a vertical divisor means an effective divisor pulled back via $\left.p_{2}\right)$. Also, denote by $x_{\alpha}^{i} \in E^{*}\left(U_{\alpha}\right), i=1,2, \ldots, n$, the dual basis of $\left\{s_{\alpha}^{i}\right\}_{i=1,2, \ldots, n}$. Then $x_{\alpha}^{1}, \ldots, x_{\alpha}^{n}$ can be seen as a coordinate of $E\left(U_{\alpha}\right)$.

The equation $\sum_{i} x_{\alpha}^{i} s_{\alpha}^{i}=0$ defines a divisor $\mathcal{D}_{\alpha}^{\prime} \subset X \times \mathbb{P}_{U_{\alpha}}\left(E_{U_{\alpha}}\right)$, which coincides with $\mathcal{D}$ if restricted to the open set $X \times \mathbb{P}_{U_{\alpha} \cap U}(E)$. We patch together the $\mathcal{D}_{\alpha}^{\prime}$ 's and get a divisor $\mathcal{D}^{\prime}$. By assumption, the divisor $\mathcal{D}^{\prime}$ has no vertical part, hence $\mathcal{D}^{\prime}=\mathcal{D}$. By construction, $\mathcal{D}^{\prime} \equiv p^{*} \mathcal{L} \otimes q^{*} \mathcal{O}_{P}(1)$, and we are done.

\section{Proof of Theorem 1.2}

This section is devoted to proving Theorem 1.2 ,

Let $\hat{P}=\mathbb{P}_{\hat{A}}\left(R \Phi_{\mathcal{P}}(E)\right), n=\operatorname{rank}(E)$ and $m=\operatorname{rank}\left(R \Phi_{\mathcal{P}}(E)\right)$. Also let $\pi: P \rightarrow$ $A$ and $\hat{\pi}: \hat{P} \rightarrow \hat{A}$ denote the natural projections, which coincide with their Albanese maps respectively. We denote by $\tilde{\mathcal{P}}$ the pull-back $(\pi \times \hat{\pi})^{*} \mathcal{P}$ of the Poincaré bundle on $A \times \hat{A}$.

By Corollary 2.2, we conclude that

$$
\left(p_{2}\right)_{*}\left(p_{1}^{*} \mathcal{O}_{P}(1) \otimes \mathcal{P}_{\pi}\right) \cong R \Phi_{\mathcal{P}}\left(\pi_{*} \mathcal{O}_{P}(1)\right) \cong R \Phi_{\mathcal{P}}(E) \cong R^{0} \Phi_{\mathcal{P}}(E)
$$

and

$$
\left(p_{1}\right)_{*}\left(p_{2}^{*} \mathcal{O}_{\hat{P}}(1) \otimes \mathcal{P}_{\hat{\pi}}\right) \cong R \Psi_{\mathcal{P}}\left(\hat{\pi}_{*} \mathcal{O}_{\hat{P}}(1)\right) \cong R \Psi_{\mathcal{P}}\left(R \Phi_{\mathcal{P}}(E)^{*}\right) \cong E^{*},
$$

where the maps $p_{1}, p_{2}$ in the first equation denote the projections from $P \times \hat{A}$ to $P$ and $\hat{A}$ respectively, and in the second equation denote the projections from $A \times \hat{P}$ to $A$ and $\hat{P}$ respectively. We identify $P$ (resp. $\hat{P}$ ) with the Hilbert scheme parametrizing the divisors in $\left\{\left|\mathcal{O}_{\hat{P}}(1) \otimes \hat{\alpha}\right| \mid \hat{\alpha} \in P i c^{0}(\hat{P})=A\right\}$ (resp. $\left\{\mid \mathcal{O}_{P}(1) \otimes\right.$ $\left.\alpha \| \alpha \in \operatorname{Pic}^{0}(P)=\hat{A}\right\}$ ), and denote by $\mathcal{Y} \subset P \times \hat{P}$ the universal family. Using Theorem 2.10 we get

$$
\mathcal{Y} \equiv p_{1}^{*} \mathcal{O}_{P}(1) \otimes p_{2}^{*} \mathcal{O}_{\hat{P}}(1) \otimes \tilde{\mathcal{P}} .
$$

Immediately from (3.1), it follows that for $x \in P$,

$$
\mathcal{Y}_{x} \equiv\left(p_{1}^{*} \mathcal{O}_{P}(1) \otimes p_{2}^{*} \mathcal{O}_{\hat{P}}(1) \otimes \tilde{\mathcal{P}}\right)_{x} \equiv \mathcal{O}_{\hat{P}}(1) \otimes \mathcal{P}_{\pi(x)} .
$$

Since $R^{i} \hat{\pi}_{*} \mathcal{O}_{\hat{P}}(1)=0$ for $i>0$ and $\hat{\pi}_{*} \mathcal{O}_{\hat{P}}(1)$ is an $I T^{0}$ sheaf, we have

$$
h^{i}\left(\hat{P}, \mathcal{O}_{\hat{P}}(1) \otimes \mathcal{P}_{a}\right)=0 \quad \text { for } i>0, a \in A,
$$

and remark that

$\diamond$ identifying a divisor in $\left|\mathcal{O}_{P}(1) \otimes \alpha\right|, \alpha \in \hat{A}$, with a point in $\hat{P}$, for every $x \in P, \mathcal{Y}_{x} \equiv \mathcal{O}_{\hat{P}}(1) \otimes \mathcal{P}_{\pi(x)}$ parametrizes all those divisors passing through $x$; and for every divisor $Y \equiv \mathcal{O}_{\hat{P}}(1) \otimes \mathcal{P}_{a}, a \in A$ (equivalently, topologically equivalent to $\mathcal{Y}_{x}$ for some $x \in P$ ), there exists a unique $y \in P$ such that $\mathcal{Y}_{y}=Y$.

We conclude that for $x, y \in P$,

$$
\mathcal{Y}_{x} \equiv \mathcal{Y}_{y} \Leftrightarrow \pi(x)=\pi(y), \mathcal{Y}_{x}=\mathcal{Y}_{y} \Leftrightarrow x=y .
$$

Also, we can write that

$$
\mathcal{Y}_{x}=\mathcal{H}_{x}+\mathcal{V}_{x} \text { and } \mathcal{V}_{x}=\mathcal{V}_{x}^{1}+\ldots+\mathcal{V}_{x}^{r}
$$


where $\mathcal{H}_{x}$ is the horizontal part (if $m=1$, then $\mathcal{H}_{x}=\emptyset$ ), $\mathcal{V}_{x}=\hat{\pi}^{*} V_{x}$ is the vertical part $\left(\mathcal{V}_{x}=\emptyset\right.$ if $\mathcal{Y}_{x}$ is irreducible), and the $\mathcal{V}_{x}^{i}=\hat{\pi}^{*} V_{x}^{i}$ 's are the reduced and irreducible vertical components (two of them may equal). Here we remark that

$\odot$ as the divisor $V_{x}$ varies continuously in $\hat{A}$, the divisor $\mathcal{Y}_{x}=\mathcal{H}_{x}+\mathcal{V}_{x}$ varies in the same topological class. Correspondingly $x$ varies continuously in $P$ by $\nabla$, so if $x$ is general, we can assume that the divisors $V_{x}^{1}, \ldots, V_{x}^{r},(-1)_{\hat{A}}^{*} V_{x}^{1}, \ldots,(-1)_{\hat{A}}^{*} V_{x}^{r}$ are distinct to each other.

Remark 3.1. Suppose that for general $x \in P, \mathcal{Y}_{x}$ is reducible. Then there exist an open set $U \subset P$ and two divisors $\mathcal{H}$ and $\mathcal{V}$ on $U \times \hat{P}$ such that for $x \in U, \mathcal{H}_{x}$ (resp. $\mathcal{V}_{x}$ ) defined above coincides with the fiber of $\mathcal{H}$ (resp. $\mathcal{V}$ ) over $x$. We also denote the closure of the two divisors in $P \times \hat{P}$ by $\mathcal{H}$ and $\mathcal{V}$. Then since $\mathcal{Y}_{x}$ is a divisor in $\hat{P}$ for every $x \in P, \mathcal{Y}$ contains no component which is the pull-back of a divisor in $P$ via the projection $P \times \hat{P} \rightarrow P$; thus $\mathcal{Y}=\mathcal{H}+\mathcal{V}$.

\subsection{Base points and the degree of $\phi$.}

Proposition 3.2. $\left|\mathcal{O}_{P}(2)\right|$ is base point free, and $\mathcal{O}_{P}(3)$ is very ample.

Proof. Fix a point $x \in P$. Since $\mathcal{Y}_{x}$ is a divisor on $P$, for a general $\alpha \in \hat{A}$, we can find $H_{\alpha} \in\left|\mathcal{O}_{P}(1) \otimes \alpha\right|$ and $H_{\alpha^{-1}} \in\left|\mathcal{O}_{P}(1) \otimes \alpha^{-1}\right|$ such that neither $H_{\alpha}$ nor $H_{\alpha^{-1}}$ is contained in $\mathcal{Y}_{x}$, which means $x$ is not contained in $H_{\alpha}+H_{\alpha^{-1}}$ by $\diamond$. Then we can see that $\left|\mathcal{O}_{P}(2)\right|$ has no base point.

Let $x \in P$, and denote by $I_{x}$ its ideal sheaf. Consider the following exact sequence:

$$
0 \rightarrow I_{x} \otimes \mathcal{O}_{P}(1) \rightarrow \mathcal{O}_{P}(1) \rightarrow \mathbb{C}(x) \rightarrow 0 .
$$

Applying $\pi_{*}$ to the sequence above we obtain an exact sequence on $A$ :

$$
0 \rightarrow \pi_{*}\left(I_{x} \otimes \mathcal{O}_{P}(1)\right) \rightarrow E \rightarrow \mathbb{C}(\pi(x)) \rightarrow 0 .
$$

Then applying $R \Phi_{\mathcal{P}}$ to the sequence above, we get

$$
0 \rightarrow R^{0} \Phi_{\mathcal{P}}\left(\pi_{*}\left(I_{x} \otimes \mathcal{O}_{P}(1)\right)\right) \rightarrow R^{0} \Phi_{\mathcal{P}}(E) \rightarrow \mathcal{P}_{\pi(x)} \rightarrow R^{1} \Phi_{\mathcal{P}}\left(\pi_{*}\left(I_{x} \otimes \mathcal{O}_{P}(1)\right)\right) \rightarrow 0
$$

and that $R^{i} \Phi_{\mathcal{P}}\left(\pi_{*}\left(I_{x} \otimes \mathcal{O}_{P}(1)\right)\right)=0$ if $i>1$, since then $R^{i} \Phi_{\mathcal{P}}(E)=R^{i} \Phi_{\mathcal{P}}(\mathbb{C}(\pi(x)))$ $=0$.

Restricting $\mathcal{Y}_{x}$ to a general fiber of $\hat{P} \rightarrow A$, it is a hyperplane, so

$$
\operatorname{rank}\left(R^{0} \Phi_{\mathcal{P}}\left(\pi_{*}\left(I_{x} \otimes \mathcal{O}_{P}(1)\right)\right)\right)=\operatorname{rank}\left(R^{0} \Phi_{\mathcal{P}}(E)\right)-1 .
$$

Also, since $\operatorname{rank}\left(\mathcal{P}_{\pi(x)}\right)=1$ we conclude that $\operatorname{codim}_{\hat{A}}\left(\operatorname{Supp}\left(R^{1} \Phi_{\mathcal{P}}\left(\pi_{*}\left(I_{x} \otimes \mathcal{O}_{P}(1)\right)\right)\right)\right)$ $\geq 1$, so $\pi_{*}\left(I_{x} \otimes \mathcal{O}_{P}(1)\right)$ is a GV-sheaf. Proposition 2.4 tells that $\pi_{*}\left(I_{x} \otimes \mathcal{O}_{P}(1)\right) \otimes E$ satisfies $I T^{0}$ and thus is CGG by Proposition 2.5. Since both the natural maps

$$
\pi_{*}\left(I_{x} \otimes \mathcal{O}_{P}(1)\right) \otimes E \rightarrow \pi_{*}\left(I_{x} \otimes \mathcal{O}_{P}(2)\right)
$$

and

$$
\pi^{*}\left(\pi_{*}\left(I_{x} \otimes \mathcal{O}_{P}(2)\right)\right) \rightarrow I_{x} \otimes \mathcal{O}_{P}(2)
$$

are surjective, $I_{x} \otimes \mathcal{O}_{P}(2)$ is CGG w.r.t. $\pi$. Note that $\mathcal{O}_{P}(1)$ is CGG w.r.t. $\pi$ since $\pi_{*} \mathcal{O}_{P}(1) \cong E$ is CGG and $\pi^{*} E \rightarrow \mathcal{O}_{P}(1)$ is surjective. Using Proposition [2.6] it follows that $I_{x} \otimes \mathcal{O}_{P}(3)$ is globally generated. Therefore, the line bundle $\mathcal{O}_{P}(3)$ is very ample. 
Lemma 3.3. Let $x, y \in P$ be two distinct points. Write that $\mathcal{Y}_{x}=\mathcal{H}_{x}+\mathcal{V}_{x}$ and $\mathcal{Y}_{y}=\mathcal{H}_{y}+\mathcal{V}_{y}$ as in (3.2). Then the following conditions are equivalent:

(a) $\left|\mathcal{O}_{P}(2)\right|$ fails to separate $x, y$;

(b) $\mathcal{H}_{x}=\mathcal{H}_{y}$ and $\operatorname{Supp}\left(V_{x}+(-1)_{\hat{A}}^{*} V_{x}\right)=\operatorname{Supp}\left(V_{y}+(-1)_{\hat{A}}^{*} V_{y}\right)$.

Proof. First we show $(a) \Rightarrow(b)$. Now suppose that $\left|\mathcal{O}_{P}(2)\right|$ fails to separate $x, y$.

We claim that $\mathcal{H}_{x}=\mathcal{H}_{y}$ if $m>1$. Indeed, otherwise, for a general $\alpha \in \hat{A}$, we can find $H_{\alpha} \in\left|\mathcal{O}_{P}(1) \otimes \alpha\right|, H_{\alpha^{-1}} \in\left|\mathcal{O}_{P}(1) \otimes \alpha^{-1}\right|$ such that $H_{\alpha} \in \mathcal{Y}_{x}$ and neither $H_{\alpha}$ nor $H_{\alpha^{-1}}$ is contained in $\mathcal{Y}_{y}$. So $H_{\alpha}+H_{\alpha^{-1}} \in\left|\mathcal{O}_{P}(2)\right|$ contains $x$ but not $y$ by $\diamond$; therefore $\left|\mathcal{O}_{P}(2)\right|$ separates $x, y$.

Let $\alpha \in V_{x}$. Then $x \in B s\left|\mathcal{O}_{P}(1) \otimes \alpha\right|$. Let $\mathcal{V}_{x}^{i}=\hat{\pi}^{*} V_{x}^{i}$ be an irreducible component of $\mathcal{Y}_{x}$. Then for any $\alpha \in V_{x}^{i}$, any $H_{\alpha} \in\left|\mathcal{O}_{P}(1) \otimes \alpha\right| \subset \mathcal{V}_{x}^{i}$ and $H_{\alpha^{-1}} \in$ $\left|\mathcal{O}_{P}(1) \otimes \alpha^{-1}\right| \subset \hat{\pi}^{*}(-1)_{\hat{A}}^{*} V_{x}^{i}$, since $x \in H_{\alpha}+H_{\alpha^{-1}}$, we have $y \in H_{\alpha}+H_{\alpha^{-1}}$, so either $H_{\alpha} \in \mathcal{Y}_{y}$ or $H_{\alpha^{-1}} \in \mathcal{Y}_{y}$. Then we conclude that $\mathcal{V}_{x}^{i} \subset \mathcal{Y}_{y}$ or $\hat{\pi}^{*}(-1)_{\hat{A}}^{*} V_{x}^{i} \subset \mathcal{Y}_{y}$ since $\mathcal{V}_{x}^{i}$ is irreducible, so it follows that

$$
\operatorname{Supp}\left(V_{x}\right) \subset \operatorname{Supp}\left(V_{y}+(-1)_{\hat{A}}^{*} V_{y}\right) \text { and } \operatorname{Supp}\left((-1)_{\hat{A}}^{*} V_{x}\right) \subset \operatorname{Supp}\left(V_{y}+(-1)_{\hat{A}}^{*} V_{y}\right) \text {. }
$$

Thus

$$
\operatorname{Supp}\left(V_{x}+(-1)_{\hat{A}}^{*} V_{x}\right) \subset \operatorname{Supp}\left(V_{y}+(-1)_{\hat{A}}^{*} V_{y}\right) .
$$

In the same way, we show that $\operatorname{Supp}\left(V_{x}+(-1)_{\hat{A}}^{*} V_{x}\right) \supset S u p p\left(V_{y}+(-1)_{\hat{A}}^{*} V_{y}\right)$, so one direction follows.

Now we show $(b) \Rightarrow(a)$, so assume $(b)$.

Note that $\pi_{*}\left(I_{x} \otimes \mathcal{O}_{P}(1)\right)$ is a GV-sheaf which is proved during the proof of Proposition 3.2. Again since the natural map

$$
\pi_{*}\left(I_{x} \otimes \mathcal{O}_{P}(1)\right) \otimes E \rightarrow \pi_{*}\left(I_{x} \otimes \mathcal{O}_{P}(2)\right)
$$

is surjective, using Remark 2.8, we conclude that $H^{0}\left(A, I_{x} \otimes \mathcal{O}_{P}(2)\right)$ is spanned by the elements in the set

$$
\begin{aligned}
\left\{e_{\alpha} \otimes f_{\alpha^{-1}} \in H^{0}\left(P, \mathcal{O}_{P}(2)\right) \mid \alpha \in \hat{A}, e_{\alpha} \in H^{0}\left(P, I_{x} \otimes \mathcal{O}_{P}(1) \otimes \alpha\right),\right. & \\
& \left.f_{\alpha^{-1}} \in H^{0}\left(P, \mathcal{O}_{P}(1) \otimes \alpha^{-1}\right)\right\} .
\end{aligned}
$$

On the other hand, reversing the argument when proving $(a) \Rightarrow(b)$, we can prove that

$$
x \in H_{\alpha}+H_{\alpha^{-1}} \Rightarrow y \in H_{\alpha}+H_{\alpha^{-1}},
$$

where $H_{\alpha} \in\left|\mathcal{O}_{P}(1) \otimes \alpha\right|, H_{\alpha^{-1}} \in\left|\mathcal{O}_{P}(1) \otimes \alpha^{-1}\right|$. Then by the fact that

$$
\left(e_{\alpha} \otimes f_{\alpha^{-1}}\right) \in\left|\mathcal{O}_{P}(1) \otimes \alpha\right|+\left|\mathcal{O}_{P}(1) \otimes \alpha^{-1}\right| \subset\left|\mathcal{O}_{P}(2)\right|,
$$

we conclude that every divisor in $\left|\mathcal{O}_{P}(2)\right|$ containing $x$ contains $y$. So this direction follows, and we are done.

Corollary 3.4. Let $x$ be a general point, and write that $\mathcal{Y}_{x}=\mathcal{H}_{x}+\mathcal{V}_{x}^{1}+\ldots+\mathcal{V}_{x}^{r}$ as in (3.2). Then the degree of $\phi$ is $2^{r}$. In particular, $\phi$ is birational if and only if for a general $x \in P, \mathcal{Y}_{x}$ is irreducible.

Proof. By $\varnothing$, we can assume the divisors $V_{x}^{1}, \ldots, V_{x}^{r},(-1)_{\hat{A}}^{*} V_{x}^{1}, \ldots,(-1)_{\hat{A}}^{*} V_{x}^{r}$ are distinct to each other. For a point $y \in P$ distinct to $x$, since $V_{x}+(-1)_{\hat{A}}^{*} V_{x}$ is reduced, using Lemma 3.3 we know that $\left|\mathcal{O}_{P}(2)\right|$ fails to separate $x$ and $y$ if and only if

$$
V_{x}+(-1)_{\hat{A}}^{*} V_{x}=V_{y}+(-1)_{\hat{A}}^{*} V_{y}
$$


equivalently,

$$
V_{y}=\left((-1)_{\hat{A}}^{\epsilon_{1}}\right)^{*} V_{x}^{1}+\ldots+\left((-1)_{\hat{A}}^{\epsilon_{r}}\right)^{*} V_{x}^{r}, \epsilon_{i} \in\{0,1\}, i=1,2, \ldots, r .
$$

On the other hand, for every choice $\epsilon_{i} \in\{0,1\}, i=1,2, \ldots, r$, since $\left((-1)_{\hat{A}}^{\epsilon_{1}}\right)^{*} V_{x}^{i}-$ $V_{x}^{i} \in P i c^{0}(\hat{A})$, there exists $\alpha \in P i c^{0}(\hat{P})$ such that

$$
\mathcal{H}_{x}+\hat{\pi}^{*}\left((-1)_{\hat{A}}^{\epsilon_{1}}\right)^{*} V_{x}^{1}+\ldots+\hat{\pi}^{*}\left((-1)_{\hat{A}}^{\epsilon_{r}}\right)^{*} V_{x}^{r} \equiv \mathcal{Y}_{x}+\alpha
$$

Thus there exists a unique $y \in P$ such that

$$
\mathcal{Y}_{y}=\mathcal{H}_{x}+\hat{\pi}^{*}\left((-1)_{\hat{A}}^{\epsilon_{1}}\right)^{*} V_{x}^{1}+\ldots+\hat{\pi}^{*}\left((-1)_{\hat{A}}^{\epsilon_{r}}\right)^{*} V_{x}^{r} .
$$

Then we conclude that $\operatorname{deg}(\phi)=2^{r}$, and the remaining assertion follows easily.

\subsection{The reducibility of the general hyperplane section.}

Theorem 3.5. For a general $x \in P$, if $\mathcal{Y}_{x}$ has a non-trivial vertical part, then $A \cong A_{1} \times A_{2}\left(A_{1}, A_{2}\right.$ maybe a point), and there exists a line bundle $L_{1}$ on $A_{1}$ and a vector bundle $E_{2}$ on $A_{2}$ such that $E \cong L_{1} \otimes E_{2}$ and that $\chi\left(A_{1}, L_{1}\right)=1$ or $\chi\left(A_{2}, E_{2}\right)=1$.

The converse is also true.

Proof. If $m=\chi(E)=1$, then $R^{0} \Phi_{\mathcal{P}}(E)$ is a line bundle on $\hat{A}$. Let $A_{1}=p t$, $A_{2}=A, L_{1}$ the trivial line bundle on $A_{1}$ and $E_{2}=E$ on $A_{2}$. Then we are done.

Now assume $m>1$. By assumption for a general $x$, we can write $\mathcal{Y}_{x}=\mathcal{H}_{x}+\mathcal{V}_{x}$ as in (3.2); correspondingly write that $\mathcal{Y}=\mathcal{H}+\mathcal{V}$ as in Remark 3.1. In the following, for every $x \in P$, the divisors $\mathcal{H}_{x}$ and $\mathcal{V}_{x}$ denote the fibers of $\mathcal{H}$ and $\mathcal{V}$ over $x$ respectively.

Let $a \in A$ be general. First we claim that for two general points $x_{1}, x_{2} \in P$ over $a, \mathcal{H}_{x_{1}} \equiv \mathcal{H}_{x_{2}}$. Indeed, for a general point $x_{0}$ fixed over $a$, we can define a morphism $\pi^{-1} a \rightarrow A$ via $x \mapsto \mathcal{H}_{x}-\mathcal{H}_{x_{0}}$ by identifying $\mathcal{H}_{x}-\mathcal{H}_{x_{0}}$ with an element in $\operatorname{Pic} c^{0}(\hat{A}) \cong A$. This map must be constant since $\pi^{-1} a$ is rational. So this assertion is true. In turn we conclude that

$$
\left|\mathcal{O}_{\hat{P}}(1) \otimes a\right|=\left|\mathcal{H}_{x}+\mathcal{V}_{x}\right|=\left|\mathcal{H}_{x}\right|+\left|\mathcal{V}_{x}\right| \text { where } x \in P \text { is a general point over } a
$$

and that $\left|\mathcal{H}_{x}\right|=\mathcal{H}_{x}$ or $\left|\mathcal{V}_{x}\right|=\mathcal{V}_{x}$ holds true due to Bertini's theorem. By semicontinuity, we conclude that both $h^{0}\left(\hat{P}, \mathcal{H}_{x}\right)$ and $h^{0}\left(\hat{P}, \mathcal{V}_{x}\right)$ are invariant as $x$ varies in $P$, and one of them is equal to $m$ and the other is equal to 1 .

Therefore, for a general $a_{0} \in A$ fixed, we can define two rational maps $\iota_{1}, \iota_{2}$ : $A \rightarrow A$ by $\iota_{1}: a \mapsto \mathcal{H}_{x}-\mathcal{H}_{x_{0}}$ and $\iota_{2}: a \mapsto \mathcal{V}_{x}-\mathcal{V}_{x_{0}}$ where $x, x_{0}$ are two general points over $a, a_{0}$ respectively, which are well defined and extend to two morphisms. Denote their images by $A_{1}$ and $A_{2}$, which are two sub-tori passing through $0 \in A$, hence subgroups of $A$.

Let $a \in A_{1} \cap A_{2}$; then $a^{-1} \in A_{1} \cap A_{2}$. We can find $x_{1}, x_{2} \in P$ such that $\iota_{1}\left(\pi\left(x_{1}\right)\right)=a, \iota_{2}\left(\pi\left(x_{2}\right)\right)=a^{-1}$. It follows that $\left|\mathcal{H}_{x_{1}}\right|=\left|\mathcal{H}_{x_{0}} \otimes a\right| \neq \emptyset$ and $\left|\mathcal{V}_{x_{2}}\right|=$ $\left|\mathcal{V}_{x_{0}} \otimes a^{-1}\right| \neq \emptyset$, and therefore,

$$
\left|\mathcal{H}_{x_{0}} \otimes a\right|+\left|\mathcal{V}_{x_{0}} \otimes\left(a^{-1}\right)\right|=\left|\mathcal{H}_{x_{1}}\right|+\left|\mathcal{V}_{x_{2}}\right| \subset\left|\mathcal{O}_{\hat{P}}(1) \otimes a_{0}\right|
$$

Note that a general divisor in $\left|\mathcal{H}_{x_{0}}\right|$ is irreducible since $a_{0}$ is general, $h^{0}\left(\hat{P}, \mathcal{H}_{x_{0}}\right)=$ $h^{0}\left(\hat{P}, \mathcal{H}_{x_{1}}\right)$, and $\mathcal{H}_{x_{0}} \sim_{\text {num }} \mathcal{H}_{x_{1}}$. So (3.3) implies that $\mathcal{H}_{x_{0}} \equiv \mathcal{H}_{x_{1}}$, thus $a=0$. Therefore, $A_{1} \cap A_{2}=\{0\}$. 
On the other hand, for general $a \in A$ and $x \in P$ over $a$, by definition it follows that

$$
\left|\mathcal{H}_{x}\right|=\left|\mathcal{H}_{x_{0}} \otimes \iota_{1}(a)\right| \neq \emptyset,\left|\mathcal{V}_{x}\right|=\left|\mathcal{V}_{x_{0}} \otimes \iota_{2}(a)\right| \neq \emptyset ;
$$

hence $\left|\mathcal{O}_{\hat{P}}(1) \otimes a\right|=\left|\mathcal{H}_{x_{0}} \otimes \iota_{1}(a)\right|+\left|\mathcal{V}_{x_{0}} \otimes \iota_{2}(a)\right|$. By $\left|\mathcal{O}_{\hat{P}}(1) \otimes a_{0}\right|=\left|\mathcal{H}_{x_{0}}\right|+\left|\mathcal{V}_{x_{0}}\right|$, we conclude that $a-a_{0}=\iota_{1}(a)+\iota_{2}(a)$; consequently $A \cong A_{1} \times A_{2}$.

Let $\hat{A}_{i}=\operatorname{Pic}^{0}\left(A_{i}\right)$ and denote by $\mathcal{P}_{i}$ the pull-back of the Poincaré bundle on $A_{i} \times \hat{A}_{i}$ via the map $A_{i} \times \hat{A} \rightarrow A_{i} \times \hat{A}_{i}$. By the analysis above we conclude that

$$
R \Psi_{\mathcal{P}}\left(\hat{\pi}_{*} \mathcal{O}_{\hat{P}}(1) \otimes a_{0}\right) \cong R^{0} \Psi_{\mathcal{P}_{1}}\left(\hat{\pi}_{*} \mathcal{O}_{\hat{P}}\left(\mathcal{H}_{x_{0}}\right)\right) \otimes R^{0} \Psi_{\mathcal{P}_{2}}\left(\hat{\pi}_{*} \mathcal{O}_{\hat{P}}\left(\mathcal{V}_{x_{0}}\right)\right)
$$

Then recalling that $\left|\mathcal{H}_{x}\right|=\mathcal{H}_{x}$ or $\left|\mathcal{V}_{x}\right|=\mathcal{V}_{x}$ holds, we conclude that at least one of $R^{0} \Psi_{\mathcal{P}_{1}}\left(\hat{\pi}_{*} \mathcal{O}_{\hat{P}}\left(\mathcal{H}_{x_{0}}\right)\right)$ and $R^{0} \Psi_{\mathcal{P}_{2}}\left(\hat{\pi}_{*} \mathcal{O}_{\hat{P}}\left(\mathcal{V}_{x_{0}}\right)\right)$ is a line bundle. Then since $R \Psi_{\mathcal{P}}\left(\hat{\pi}_{*} \mathcal{O}_{\hat{P}}(1) \otimes a_{0}\right) \cong t_{-a_{0}}^{*} E^{*}$ where $t_{-a_{0}}$ is the translation by $-a_{0}$, one direction is completed.

The converse assertion is obvious, so this theorem is true.

3.3. The map $\phi$. We distinguish the two cases $m=1$ and $m>1$.

Case $m=1$. In this case, for every $x \in P, \mathcal{Y}_{x}$ is a divisor $V_{x}$ on $\hat{A}$. Hence $\phi$ is surely not birational by Corollary 3.4, and $E=R \Psi_{\mathcal{P}}\left(\mathcal{O}_{\hat{A}}(V)\right)^{*}$ for some ample divisor $V$ on $\hat{A}$. Find a suitable $a_{0} \in A$ such that $\mathcal{O}_{\hat{A}}(V) \otimes a_{0}$ is symmetric. Then its Fourier-Mukai transform $R \Psi_{\mathcal{P}}\left(\mathcal{O}_{\hat{A}}(V) \otimes a_{0}\right) \cong t_{-a_{0}}^{*} E^{*}$ is a $(-1)_{A}$-invariant sheaf. Replacing $E$ by $t_{-a_{0}}^{*} E$, we can assume $E$ is a $(-1)_{A}$-invariant sheaf, so $(-1)_{A}$ induces an action $(-1)_{A}^{*}$ on $H^{0}(A, E \otimes E)$. Note that $H^{0}\left(P, \mathcal{O}_{P}(2)\right) \cong H^{0}\left(A, S^{2} E\right)$. By Lemma 2.9, $(-1)_{A}^{*}$ is the identity on $H^{0}\left(A, S^{2} E\right)$, hence $\phi$ factors through an involution $\sigma$ making the following commutative diagram hold:

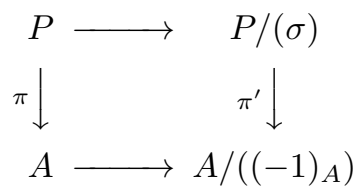

In particular, if $A$ is simple, then Corollary 3.4 gives that $\operatorname{deg}(\phi)=2$ if $\operatorname{dim}(A)>$ 1 since then a general $V_{x}$ is irreducible, and $\operatorname{deg}(\phi)=2^{n}$ if $\operatorname{dim}(A)=1$ since then $\operatorname{deg}\left(V_{x}\right)=\operatorname{rank}(E)=n$.

Case $m>1$. In this case, using Corollary 3.4, it immediately follows that $\phi$ is not birational if and only if for a general $x \in P, \mathcal{Y}_{x}$ is reducible.

If $A$ is simple and $\phi$ is not birational, applying Theorem 3.5 we conclude that $A \cong A_{1} \times A_{2}$ and hence either $A_{1}$ or $A_{2}$ is a point. So we can assume $A_{1}=A, L_{1}$ is a line bundle on $A_{1}$ such that $\left(A, L_{1}\right)$ is a principal polarization, $A_{2}=p t$ and $E_{2}$ is a vector bundle of rank $>1$. Therefore $P \cong A \times \mathbb{P}^{n-1}$; then reducing to the case when $m=1$, we can show $\phi$ is of degree 2 .

In conclusion, we finally proved Theorem 1.2

3.4. The degree of $\phi$ when $A$ is not simple. When $A$ is not simple, to study the degree of $\phi$, it suffices to consider the case when $m=1$; i.e., $R \Phi_{\mathcal{P}}(E)$ is quasi-isomorphic to a line bundle. This is completely clear thanks to the following classical result. 
Theorem 3.6 ([1], Sec. 3.4). Let $A$ be an Abelian variety and $D$ be an ample divisor on $A$. Then the linear system $|D|$ can be written as $|D|=\left|D_{1}\right|+D_{2}+\ldots+D_{r}$, where:

(i) $\left|D_{1}\right|$ is the moving part;

(ii) $A \cong A_{1} \times A_{2} \times \ldots \times A_{r}$, where $A_{i}, i=2,3, \ldots, r$, is simple;

(iii) for $i=1,2, \ldots, r, D_{i} \equiv p_{i}^{*} B_{i}$, where $B_{i}$ is a divisor on $A_{i}$, and for $i=$ $2, \ldots, r,\left(A_{i}, B_{i}\right)$ is a principal polarization.

Moreover, if $\operatorname{dim}\left(A_{1}\right)>1$, then a general element in $\left|B_{1}\right|$ is irreducible.

Corollary 3.7. Assume that $R \Phi_{\mathcal{P}}(E)=\mathcal{O}_{\hat{A}}(-\hat{D})$ for some ample divisor $\hat{D}$ on $\hat{A}$ and that the linear system $|\hat{D}|=\left|\hat{D}_{1}\right|+\hat{D}_{2}+\ldots+\hat{D}_{r}$ as in Theorem 3.6, where $\hat{A} \cong \hat{A}_{1} \times \hat{A}_{2} \times \ldots \times \hat{A}_{r}$ and $\hat{D}_{i}=p_{i}^{*} \hat{B}_{i}$. Then we have:

(1) if $\hat{A}_{1}$ is an elliptic curve, then $\operatorname{deg}(\phi)=2^{\operatorname{deg}\left(\hat{B}_{1}\right)+r-1}$;

(2) if $\operatorname{dim}\left(\hat{A}_{1}\right)>1$, then $\operatorname{deg}(\phi)=2^{r}$.

Proof. For $a \in A$, there exists $\alpha \in \hat{A}$ such that $\mathcal{O}_{\hat{A}}(\hat{D}) \otimes \mathcal{P}_{a} \equiv t_{\alpha}^{*} \hat{D}$; hence

$$
\left|\mathcal{O}_{\hat{A}}(\hat{D}) \otimes \mathcal{P}_{a}\right|=\left|t_{\alpha}^{*} \hat{D}_{1}\right|+t_{\alpha}^{*} \hat{D}_{2}+\ldots+t_{\alpha}^{*} \hat{D}_{r} .
$$

For $x \in P$, the divisor $\mathcal{V}_{x} \in\left|\mathcal{O}_{\hat{A}}(\hat{D}) \otimes \mathcal{P}_{\pi(x)}\right|$. Then our assertions follow from Corollary 3.4

3.5. Applications. The results and the ideas of the proof probably find their applications when considering the morphism defined by the square of a line bundle on an irregular variety. Here we prove that

Proposition 3.8. Let $a: X \rightarrow A$ be a generically finite morphism to an Abelian variety and $V$ a Cartier divisor on $X$ such that $\mathcal{O}_{X}(V)$ is full w.r.t. a (see Definition 2.3). Then $\mathcal{O}_{X}(V)$ is $C G G$ at general points of $X$ w.r.t. a, and the linear system $|2 V|$ defines a generically finite map. In particular, the divisor $V$ is big.

Proof. If $a^{*}: \operatorname{Pic}^{0}(A) \rightarrow P i c^{0}(X)$ is not an embedding, then we get a factorization

$$
a=\iota \circ a^{\prime}: X \rightarrow A^{\prime} \rightarrow A,
$$

where $A^{\prime}$ is the Abelian variety with dual $\operatorname{Pic}^{0}\left(A^{\prime}\right)=a^{*} \operatorname{Pic}^{0}(A) \subset P i c^{0}(X)$, and $\iota: A^{\prime} \rightarrow A$ arises from the dual map $a^{*}: \operatorname{Pic}^{0}(A) \rightarrow a^{*} \operatorname{Pic}^{0}(A)=\operatorname{Pic}^{0}\left(A^{\prime}\right)$. Note that $\iota^{*}: \operatorname{Pic}^{0}(A) \rightarrow \operatorname{Pic} c^{0}\left(A^{\prime}\right)$ is finite and surjective, and hence maps an open set to an open set. Since $H^{0}\left(X, F \otimes a^{*} \alpha\right)=H^{0}\left(X, F \otimes a^{* *} \iota^{*} \alpha\right)$ for a sheaf $F$ on $X$ and $\alpha \in \hat{A}$, we conclude that for a sheaf $F$ on $X$,

$$
F \text { is } C G G \text { w.r.t. } a \Leftrightarrow F \text { is } C G G \text { w.r.t. } a^{\prime}
$$

and

$$
F \text { is full w.r.t. } a \Leftrightarrow F \text { is full w.r.t. } a^{\prime} \text {. }
$$

Then we consider the map $a^{\prime}$ instead, so we can assume that $a^{*}: \operatorname{Pic}^{0}(A) \rightarrow$ $\operatorname{Pic}^{0}(X)$ is injective in the following.

Consider the line bundle $\mathcal{L}:=p_{1}^{*} \mathcal{O}_{X}(V) \otimes \mathcal{P}_{a}$ on $X \times \hat{A}$. Put

$$
\hat{P}=\operatorname{Proj}_{\mathcal{O}_{A}}\left(\bigoplus S^{n}\left(\left(p_{2}\right)_{*} \mathcal{L}\right)^{*}\right) .
$$


Then there exists an open set $U$ of $\hat{P}$ that parametrizes the divisors in $\mid \mathcal{O}_{X}(V) \otimes$ $\alpha \mid, \alpha \in \hat{A}$. We can find an open set $U_{0} \subset \hat{A}$ and a section $s: U_{0} \rightarrow U$. Identifying $U_{0}$ with $s\left(U_{0}\right)$, we get a universal divisor

$$
\mathcal{Y} \subset X \times U_{0}
$$

and denote its closure in $X \times \hat{A}$ by $\mathcal{V}$. Then $\mathcal{V}_{\alpha} \equiv \mathcal{O}_{X}(V) \otimes \alpha$ for $\alpha \in U_{0}$.

As in 2], Sec. 5, take a general $\alpha_{0} \in U_{0}$ and define a map

$$
f_{\alpha_{0}}: \operatorname{Pic}^{0}(A) \rightarrow \operatorname{Pic}^{0}(X), \alpha \mapsto \mathcal{O}_{X}\left(\mathcal{V}_{\alpha}-\mathcal{V}_{\alpha_{0}}\right)
$$

which extends to a morphism. By rigidity,

$$
f:=f_{\alpha_{0}}-f_{\alpha_{0}}(0): P i c^{0}(A) \rightarrow P i c^{0}(X)
$$

is a homomorphism.

By definition, the image of $f$ coincides with $a^{*} P i c^{0}(A)$. Identifying $\hat{A}=\operatorname{Pic}^{0}(A)$ with $a^{*} P i c^{0}(A)$, we have $f=i d_{\hat{A}}$. Then arguing as in Section 5.3 in [2], we prove

$$
\mathcal{V} \equiv p_{1}^{*} \mathcal{O}_{X}(V) \otimes \mathcal{P}_{a} \otimes p_{2}^{*} \mathcal{O}_{\hat{A}}\left(\mathcal{V}_{p}\right)
$$

where $p \in X$ is a point mapped to $0 \in A$ via $a$. Note that for $x \in X, \mathcal{V}_{x}=\{\alpha \in$ $\left.\hat{A} \mid x \in \mathcal{V}_{\alpha}\right\}$, and for two distinct points $x, y \in X$, if $a(x) \neq a(y)$, then $\mathcal{V}_{x} \neq \mathcal{V}_{y}$ since they are not even linearly equivalent. Since the map $a$ is finite, considering the universal divisor $\mathcal{V}$ and arguing as in the proof of Lemma 3.3. we can show that $|2 V|$ defines a generically finite map.

Identifying $\hat{A}$ with a family of divisors on $X$ via $\alpha \mapsto \mathcal{V}_{\alpha}$, then for a general point $x \in X, \mathcal{V}_{x}$ is a divisor on $\hat{A}$ which parametrizes the divisors passing through $x$. So it is easy to conclude that $\mathcal{O}_{X}(V)$ is CGG at general points of $X$.

\section{ACKNOWLEDGEMENTS}

The author would like to thank Dr. Hao Sun for his discussions and help in improving Theorem 1.2. The author also expresses his gratitude to two anonymous referees for their many useful suggestions.

\section{REFERENCES}

[1] Christina Birkenhake and Herbert Lange, Complex abelian varieties, 2nd ed., Grundlehren der Mathematischen Wissenschaften [Fundamental Principles of Mathematical Sciences], vol. 302, Springer-Verlag, Berlin, 2004. MR2062673(2005c:14001)

[2] Miguel Angel Barja, Martí Lahoz, Juan Carlos Naranjo, and Giuseppe Pareschi, On the bicanonical map of irregular varieties, J. Algebraic Geom. 21 (2012), no. 3, 445-471, DOI 10.1090/S1056-3911-2011-00565-1. MR2914800

[3] Fabrizio Catanese, Ciro Ciliberto, and Margarida Mendes Lopes, On the classification of irregular surfaces of general type with nonbirational bicanonical map, Trans. Amer. Math. Soc. 350 (1998), no. 1, 275-308, DOI 10.1090/S0002-9947-98-01948-5. MR1422597 (98h:14043)

[4] Jungkai A. Chen and Christopher D. Hacon, Pluricanonical systems on irregular 3-folds of general type, Math. Z. 255 (2007), no. 2, 343-355, DOI 10.1007/s00209-006-0028-9. MR2262735(2008i:14059)

[5] Olivier Debarre, On coverings of simple abelian varieties (English, with English and French summaries), Bull. Soc. Math. France 134 (2006), no. 2, 253-260. MR2233707 (2007j:14063)

[6] Christopher D. Hacon, A derived category approach to generic vanishing, J. Reine Angew. Math. 575 (2004), 173-187, DOI 10.1515/crll.2004.078. MR2097552(2005m:14026)

[7] Robert Lazarsfeld, Positivity in algebraic geometry. I, II. Ergebnisse der Mathematik und ihrer Grenzgebiete, vols. 48, 49, Springer-Verlag, Berlin, 2004. MR2095471 (2005k:14001a). MR2095472(2005k:14001b) 
[8] Shigeru Mukai, Duality between $D(X)$ and $D(\hat{X})$ with its application to Picard sheaves, Nagoya Math. J. 81 (1981), 153-175. MR607081 (82f:14036)

[9] Akira Ohbuchi, Some remarks on ample line bundles on abelian varieties, Manuscripta Math. 57 (1987), no. 2, 225-238, DOI 10.1007/BF02218082. MR871633 (87m:14051)

[10] Giuseppe Pareschi and Mihnea Popa, Regularity on abelian varieties III: relationship with generic vanishing and applications, Grassmannians, moduli spaces and vector bundles, Clay Math. Proc., vol. 14, Amer. Math. Soc., Providence, RI, 2011, pp. 141-167. MR2807853 (2012h:14113)

[11] Giuseppe Pareschi and Mihnea Popa, GV-sheaves, Fourier-Mukai transform, and generic vanishing, Amer. J. Math. 133 (2011), no. 1, 235-271, DOI 10.1353/ajm.2011.0000. MR2752940(2012e:14043)

[12] S. Ramanan, Ample divisors on abelian surfaces, Proc. London Math. Soc. (3) 51 (1985), no. 2, 231-245, DOI 10.1112/plms/s3-51.2.231. MR794112 (87d:14034)

College of Mathematics and Information Sciences, Shaanxi Normal University, Xi'an 710062, People's Republic of China

E-mail address: lzhpkutju@gmail.com 